\title{
Expression of Rv2031c-Rv2626c fusion protein in Mycobacterium smegmatis enhances bacillary survival and modulates innate immunity in macrophages
}

\author{
HONG JIANG $^{1 *}$, TAI-LAI LUO ${ }^{2 *}$, JIAN KANG $^{3}$, ZHI-KAI XU ${ }^{3}$ and LI-MEI WANG ${ }^{3}$ \\ ${ }^{1}$ Center for Infectious Diseases, Tangdu Hospital, The Fourth Military Medical University, Xi'an, Shaanxi 710038; \\ ${ }^{2}$ Naval Medical Research Institute, Shanghai 200433; ${ }^{3}$ Department of Microbiology, School of Basic \\ Medicine, The Fourth Military Medical University, Xi'an, Shaanxi 710032, P.R. China
}

Received March 13, 2017; Accepted November 21, 2017

DOI: $10.3892 / \mathrm{mmr} .2018 .8758$

\begin{abstract}
Dormancy-associated antigens encoded by the dormancy survival regulon (DosR) genes are required for survival of Mycobacterium tuberculosis (Mtb) in macrophages. However, mechanisms underlying survival of Mtb in macrophages remains to be elucidated. A recombinant Mycobacterium smegmatis strain (rMs) expressing a fusion protein of two dormancy-associated antigens Rv2031c and Rv2626c from Mtb was constructed in the present study. In an in vitro culture, growth rate of rMs was lower compared with Ms. A total of $24 \mathrm{~h}$ following infection of murine macrophages with rMs or Ms, percentage of viable cells decreased and the number of bacteria in viable cells increased compared with Ms, demonstrating that virulence and intracellular survival of rMs were enhanced. Compared with macrophages infected with Ms, necrosis of macrophages infected with rMs was increased, while apoptosis was inhibited. Macrophages infected with rMs secreted more interferon- $\gamma$ and interleukin- 6 , but fewer nitric oxide and tumor necrosis factor- $\alpha$, compared with macrophages infected with Ms. The present study demonstrated that the fusion protein composed of dormancy-associated antigens Rv2031c and Rv2626c in Ms serves a physiological function of a dormancy-associated antigen and modulates innate immunity of host macrophages, therefore favoring intracellular bacillary survival.
\end{abstract}

Correspondence to: Dr Li-Mei Wang, Department of Microbiology, School of Basic Medicine, The Fourth Military Medical University, 169 Changle West Road, Xi'an, Shaanxi 710032, P.R. China

E-mail: limwang79@hotmail.com

${ }^{*}$ Contributed equally

Key words: Mycobacterium tuberculosis, Mycobacterium smegmatis, Rv2031c, Rv2626c, macrophage

\section{Introduction}

Tuberculosis (TB) is a worldwide public health concern caused by Mycobacterium tuberculosis (Mtb), and $\sim$ a third of the world's population is latently infected with Mtb (1). The majority of infected people do not present symptoms immediately, but $\sim 10 \%$ of those people develop an overt disease later in their lives. Latently infected individuals represent a reservoir of infection and potential reactivation of TB can be a source of transmission (2). Macrophages represent a primary target of infection and the most frequently infected cell type by Mtb in host individuals. The initial interaction between macrophages and Mtb determines the outcome of infection, but the mechanism underlying the interaction between macrophages and Mtb remains to be elucidated (3).

It has been demonstrated that during the latency period, Mtb remains in a dormant or non-replicating state, and the dormancy survival regulon (DosR), composed of 48 co-regulated genes, is necessary for survival of dormant Mtb (4). However, the role of dormancy-associated antigens in mediating interactions between Mtb and macrophages remains to be elucidated. The $16-\mathrm{kDa} \alpha$-crystallin protein, (Rv2031c), also known as hspX, acr and Hsp16.3, is a predominant protein produced by Mtb, accounting for up to $25 \%$ of all proteins expressed during dormancy of Mtb (5). Rv2031c can be identified by mass spectrometry in culture filtrates, membrane protein fractions and whole cell lysates of Mtb (6). Rv2031c has been hypothesized to enhance long-term stability of proteins and cell structures, which in turn aids in maintaining long-term survival of Mtb (7). Rv2626c is a hypoxic response protein encoded by Mtb open reading frame $R v 2626 c$. Rv2626c is also one of the highly expressed proteins by Mtb in hypoxic conditions and can be identified in culture filtrates and lysates of Mtb (8), but the role served by Rv2626c remains to be elucidated.

In the present study, a fusion protein of Rv2031c and Rv2626c was expressed in a non-pathogenic, fast growing Mycobacterium semegmatis (Ms), to describe the physiological function of the fusion protein in mycobacteria and to investigate its immuno-modulatory functions in macrophages. 


\section{Materials and methods}

Strains of bacteria, media and growth conditions. The Ms strain $\mathrm{MC}^{2} 155$ was purchased from the American Type Culture Collection (ATCC; Manassas, VA, USA). Bacillus Calmette-Guérin (BCG) was obtained from Shaanxi Research Institute for Tuberculosis Control and Prevention (Shaanxi, China). Mycobacterial strains Ms mc ${ }^{2} 155$ and BCG were cultured in Middle brook 7H9 broth and 7H10 agar (Difco Laboratories, Detroit, MI, USA) containing albumin dextrose complex [5 $\mathrm{g}$ bovine serum albumin (Sigma-Aldrich; Merck KGaA, Darmstadt, Germany) $2 \mathrm{~g}$ glucose and $0.85 \mathrm{~g}$ $\mathrm{NaCl} / 1], 0.5 \%$ volume/volume (v/v) glycerol and $0.05 \%$ Tween-80. E. coli DH5a (Takara Biotechnology Co., Ltd., Dalian, China) was cultured in Luria Bertani media (Takara Biotechnology Co., Ltd.). Both E. coli and mycobacteria were cultured at $37^{\circ} \mathrm{C}$ in an incubator, with agitation. Hygromycin (Sigma-Aldrich; Merck KGaA) was added to certain treatment groups: $50 \mathrm{mg} / \mathrm{ml}$ to the E. coli culture and $15 \mathrm{mg} / \mathrm{ml}$ to the mycobacteria culture. All recombinant (r)Ms strains were cultured in the presence of $15 \mathrm{mg} / \mathrm{ml}$ hygromycin.

Construction of rMs strain expressing rv2031c-rv2626c fusion protein. In order to construct a rMs strain expressing Rv2031c-Rv2626 fusion protein, an expression vector was constructed by cloning Rv2031c and Rv2626 genes into the E. coli-Mycobacterium shuttle vector pDE22 (constructed in-house) (Fig. 1) (9). Primers were designed based on nucleotide sequences of Rv2031c and Rv2626c genes from the Mtb H37Rv strain. Rv2031 gene was amplified using the following primers: 5'-CGGGATCCATGGCCACCACCCTTC-3' (BamHI site underlined; forward) and 5'-AGCGATATCGTTGGTGGA CCGG-3' (EcoRV site underlined; reverse). Rv2626c gene was amplified using the following primers: 5'-AGCGATATCGGT GGCGGTAGCGGCGGTGGCTCCGGCGGTGGCAGCGGT GGCGGTAGCACCACCGCACGC-3' (EcoRV site underlined; forward) and 5'-AGCAAGCTTCTAGCTGGCGAGGGC-3' (HindIII site underlined; reverse). A 48-base pair sequence encoding a hydrophobic linker (italics) was added in the linker sequence between the 3 'end of $R v 2031 c$ and the 5 'end of $R v 2626 c$ to ensure the correct folding of each protein. The following thermocycling conditions were used for the polymerase chain reaction (PCR): Following an initial denaturation at $95^{\circ} \mathrm{C}$ for $1 \mathrm{~min}, 30$ cycles of $94^{\circ} \mathrm{C}$ for $45 \mathrm{sec}, 65^{\circ} \mathrm{C}$ for $45 \mathrm{sec}, 72^{\circ} \mathrm{C}$ for $50 \mathrm{sec}$; and a final extension at $72^{\circ} \mathrm{C}$ for $5 \mathrm{~min}$. Sequences of all resulting PCR products were validated by Sunny Biotechnology Co. (Westmont, IL, USA) and the correct sequences were identical to those reported by the GeneBank database. PCR products corresponding to each gene were cloned into the multiple cloning site region of the shuttle vector pDE22 using restriction endonucleases. The resulting recombinant plasmids were transfected into competent Ms cells by electroporation, and the transformed Ms were selected on solid 7H10 agar containing hygromycin $(50 \mu \mathrm{g} / \mathrm{ml}$; Sigma-Aldrich, Merck KGaA) for 3 days. Following selection, hygromycin-resistant colonies were transferred to fresh middlebrook 7H9 media with $15 \mathrm{mg} / \mathrm{ml}$ hygromycin. The optical density was measured at a wavelength of $600 \mathrm{~nm}\left(\mathrm{OD}_{600 \mathrm{~mm}}\right)$ and when a colony reached $\mathrm{OD}_{600 \mathrm{~nm}}=1.0$, cells were incubated at $42^{\circ} \mathrm{C}$ for $4 \mathrm{~h}$.
A total of $2 \mathrm{ml}$ of each cell culture was harvested by centrifuging at $8,000 \mathrm{x} \mathrm{g}$ for $20 \mathrm{~min}$ at room temperature. The supernatant was transferred into an Amicon ultrafiltration tube with a membrane NMWL of $10 \mathrm{kDa}$, centrifuged at $3,000 \mathrm{x} \mathrm{g}$ at $4^{\circ} \mathrm{C}$ until approximately $10 \mu \mathrm{l}$ fluid remained in the chamber, before adding $100 \mu \mathrm{l}$ sterile-distilled water and centrifuged again at $3,000 \mathrm{xg}$ at $4^{\circ} \mathrm{C}$ until $\sim 10 \mu \mathrm{l}$ fluid remained in the chamber. After centrifugation, the upper chamber of the unltrfiltraion tube was transferred to a fresh mirofuge tube and centrifuged at $3,000 \times \mathrm{g}$ at $4^{\circ} \mathrm{C}$ for $2 \mathrm{~min}$, and the volume in the tube was determined and added to an equal volume of $2 \mathrm{X}$ SDS-PAGE sample buffer. The cell pellet was resuspended in $1 \mathrm{ml}$ sterile-distilled water and re-centrifuged at $8,000 \mathrm{x} \mathrm{g}$ for $10 \mathrm{~min}$ at room temperature, then resuspended in $100 \mu \mathrm{l}$ sterile-distilled water and sonicated on ice using 4 pulses for $15 \mathrm{sec}$ on maximum output, then $100 \mu 12 \mathrm{X}$ SDS-PAGE sample buffer was added. A total of $10 \mu l$ samples were loaded onto an 12\% SDS-PAGE gel and proteins from gel were electrotransferred to a polyvinylidene difluoride membrane (pore size $0.2 \mu \mathrm{m}$; Bio-Rad Laboratories, Inc., Hercules, CA, USA) at $70 \mathrm{~V}$ for $2 \mathrm{~h}$ at $4^{\circ} \mathrm{C}$ in Tris-Glycine transfer buffer composed of $25 \mathrm{mM}$ Tris, $192 \mathrm{mM}$ glycine and $20 \%$ methanol at $\mathrm{pH} 8.3$.

For immunoblotting, non-specific binding sites were blocked with PBS containing 5\% non-fat milk for $1 \mathrm{~h}$ at room temperature. Blocked membranes were incubated overnight at $4^{\circ} \mathrm{C}$ in PBS with mouse anti-Rv2031c monoclonal antibody (cat. no. ab64786, dilution 1:500), mouse anti-Rv2626c monoclonal antibody (cat. no. ab64786, dilution 1:500) (both from Abcam, Cambridge, UK). Washed membranes were incubated $1 \mathrm{~h}$ at room temperature with IRDye $800 \mathrm{CW}$ anti-mouse antibody (1:5,000; LI-COR Bioscience, Lincoln, NE, USA), washed and immunodetection was performed using an ODYSSEY Infrared Imaging system (LI-COR Bioscience). Following screening, positive recombinant Ms strains were classified as rMs.

In vitro growth kinetics of rMs. To examine the growth pattern of rMs, rMs and Ms strains were cultured until late exponential phase, diluted to $\mathrm{OD}_{600 \mathrm{~nm}}=0.2$ and cultured in Middlebrook 7H9. Growth curves were generated by measuring alterations in $\mathrm{OD}_{600 \mathrm{~nm}}$ over time for $57 \mathrm{~h}$.

Macrophage infection. RAW264.7 murine macrophages (provided by Dr. Shi CH, the Fourth Military Medical University, Xi'an, China) were cultured at $37^{\circ} \mathrm{C}$ in $5 \% \mathrm{CO}_{2}$ in Dulbecco's modified Eagle's medium (DMEM) supplemented with 10\% (v/v) fetal bovine serum (both from Gibco; Thermo Fisher Scientific,Inc.), $1 \%$ L-glutamin and antibiotics $[60 \mathrm{mg} / \mathrm{ml}$ penicillin G sodium, $50 \mathrm{mg} / \mathrm{ml}$ streptomycin sulphate and $30 \mathrm{mg} / \mathrm{ml}$ gentamycin sulphate, purchased from Leagene Co., Beijing, China; www.leagene.bioon.com.cn). Cells were seeded in 6-well plates at a density of $0.5 \times 10^{5}$ cells/well and used for infection $24 \mathrm{~h}$ later. Exponentially growing bacteria cultured in the presence of $15 \mathrm{mg} / \mathrm{ml}$ hygromycin were pelleted, washed and resuspended in DMEM (without antibiotics) to $\mathrm{OD}_{600 \mathrm{~nm}}=1.0$. Single cell suspensions of $\mathrm{rMs}$ and Ms strains were obtained by passing cultures $\sim 5-6$ times through 26 gauge needles. Bacillary viability was assessed at each step by colony-forming unit (CFU) counts. Equal numbers of each strain were used to infect macrophages at 
a multiplicity of infection $(\mathrm{MOI})=10: 1$, selected based on pilot infections (data not shown) with multiple MOIs that we performed with cell lines used in the present study. Following incubation with bacteria for $4 \mathrm{~h}$, non-phagocytosed bacteria were washed off using PBS. Cells were washed with PBS and post-infection CFU counts were determined by lysing infected cells. Subsequently, complete DMEM containing gentamycin (Gibco; Thermo Fisher Scientific, Inc.) was added to eliminate extracellular bacteria. Infected cells were transferred to fresh DMEM and incubated for $24 \mathrm{~h}$ at $37^{\circ} \mathrm{C}$ with $5 \% \mathrm{CO}_{2}$. Following the incubation, $20 \mu \mathrm{l}$ MTT (Sigma-Aldrich; Merck $\mathrm{KGaA}$ ) was added to each sample. Following $4 \mathrm{~h}$ of incubation with MTT, dimethyl sulfoxide was added and the samples were incubated at $37^{\circ} \mathrm{C}$ for $10 \mathrm{~min}$. Each sample was observed under an optical microscope and the absorbance was measured at a wavelength of $490 \mathrm{~nm}$ on a microplate reader (Omega Bio-Tek, Inc., Norcross, GA, USA). CFU counts were performed at 3, 6, 12 and $21 \mathrm{~h}$ post infection by lysing $1 \times 10^{3}$ infected cells with $0.1 \%$ Triton X-100 (Sigma-Aldrich; Merck KGaA) followed by dilution plating on Middlebrook 7H10 agar, the results were expressed as $\log _{10} \mathrm{CFU} / 10^{3}$ cells.

Apoptosis and necrosis of macrophages. RAW264.7 murine macrophages $\left(10^{5}\right.$ cells) were left uninfected as controls or infected with Ms or rMs at 10:1 MOI for $24 \mathrm{~h}$. Macrophages were subsequently removed from plates using accutase solution (Sigma-Aldrich; Merck KGaA), washed twice in ice-cold PBS and stained with propidium iodide (PE)-conjugated Annexin V and 7-aminoactinomycin D (7AAD), according to the manufacturer's protocol (BD Biosciences). Cells were fixed in PBS containing 5\% paraformaldehyde (Sigma-Aldrich; Merck $\mathrm{KGaA}$ ) for $20 \mathrm{~min}$ at room temperature and analyzed with a FACSCanto II cytometer and FACSDiva software (version 6.1.2; BD Biosciences). Apoptosis was expressed as the percentage of Annexin V-positive 7-AAD-negative cells, and necrosis was expressed as the percentage of Annexin $\mathrm{V}$ and 7-AAD-double positive cells.

Cytokine and nitrite assays. Levels of interferon- $\gamma$ (IFN- $\gamma)$, tumor necrosis factor- $\alpha$ (TNF- $\alpha$ ) and interleukin (IL)- 6 in macrophage culture supernatants $24 \mathrm{~h}$ post infection were quantitated with a mouse IFN- $\gamma$ ELISA development kit (cat. no. 3321-1H-6), mouse TNF- $\alpha$ development kit (cat. no. 3511-1H-6) and mouse IL-6 ELISA development kit (cat. no. 3361-1H-6) according to the manufacturer's instructions. All the kits were purchased from Mabtech AB, Stockholm, Sweden. Estimation of nitric oxide (NO) levels was performed using the Griess test. Equal volumes of cell culture supernatants were transferred in duplicate into 96-well culture plates and mixed with an equal volume of Griess reagent, composed of $1 \%$ weight/volume (w/v) sulphanilamide, $0.1 \%$ $(\mathrm{w} / \mathrm{v})$ napthyl-ethylenediamine hydrochloride and $2.5 \%(\mathrm{v} / \mathrm{v})$ $\mathrm{H}_{3} \mathrm{PO}_{4}$. Following incubation at room temperature for $5 \mathrm{~min}$, the absorbance was measured at a wavelength of $540 \mathrm{~nm}$ using an Ultra Microplate Reader (Omega Bio-Tek, Inc., Norcross, GA, USA). The concentration of nitrate was calculated using a $\mathrm{NaNO}_{2}$ standard curve.

Statistical analysis. All experiments were performed in triplicate. Differences between groups were analyzed by one-way

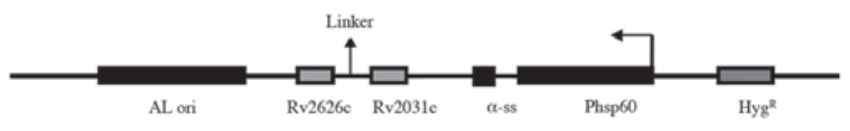

Figure 1. Schematic representation of Rv2031c and Rv2626c fusion construct generated in the Escherichia coli-Mycobacterium shuttle plasmid pDE22 under the control of the constitutive hsp60 promoter.
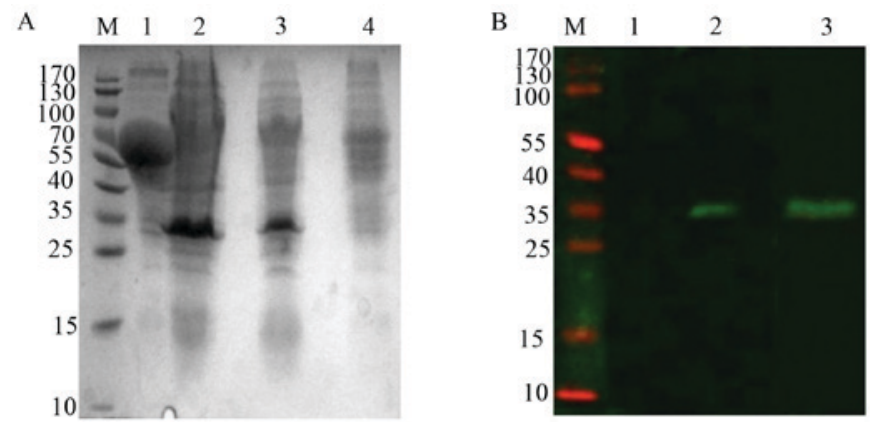

Figure 2. Expression of Rv2031c-Rv2626c fusion protein in rMs. (A) Western blot analysis of expression of Rv2031c and Rv2626c fusion protein in rMs induced by heat shock at $42^{\circ} \mathrm{C}$. Lane $\mathrm{M}$, molecular weight of standard protein markers; lane 1, supernatant of rMs culture; lanes 2 and 3, cell lysates of rMs; and lane 4, the cell lysate of Ms. (B) Western blot analysis of the fusion protein by anti-Rv2031c mAb and anti-Rv2626c mAb. Lane M, pre-stained protein markers; lane 1, the cell lysate of Ms; lane 2, cell lysate of rMs stained with an anti-Rv2031 mAb; and lane 3, cell lysate of rMs stained with anti-Rv2626c mAb. mAb, monoclonal antibody; rMs, recombinant Mycobacterium smegmatis.

analysis of variance using SPSS software (version 15.0; SPSS, Inc., Chicago, IL, USA), followed by the Fisher-Tukey least significant difference post hoc test. Data are presented as the mean \pm standard deviation. $\mathrm{P}<0.05$ was considered to indicate a statistically significant difference.

\section{Results}

Expression of Rv2031c-Rv2626c fusion protein in rMs. Total proteins from whole cell lysates of rMs and Ms strains were obtained following induction at $42^{\circ} \mathrm{C}$. Western blot analysis revealed that a specific expression band $\sim 34 \mathrm{kDa}$, corresponding to the combined molecular weight of Rv2031c (16.3 kDa) and Rv2626c (16 kDa), was present in the cell lysate of rMs, and absent in Ms cells (Fig. 2A). The above results were further confirmed by western blot analysis with anti-Rv2031c and anti-Rv2626 monoclonal antibodies, which indicated that both Rv2031c and Rv2626c were correctly folded in the Rv2031c-Rv2626c fusion protein (Fig. 2B).

Intracellular and in vitro growth characteristics of $r M s$. In order to determine whether the expression of Rv2031c-Rv2626c fusion protein in rMs alters growth characteristics of the strain, growth rates of rMs and Ms in vitro cultures were identified by $\mathrm{OD}_{600 \mathrm{~nm}}$ measurement. When log-phase cultures of rMs and Ms were allowed to grow to saturation and were equivalently diluted, the duration of the growth lag phase of rMs was significantly prolonged in rMs compared with Ms $(\mathrm{P}<0.05)$. The final log-phase rates were not significantly different (Fig. 3A). To identify 
A

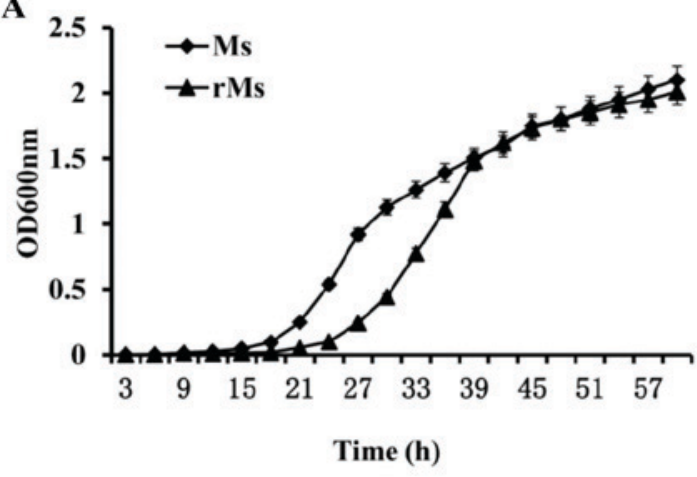

B

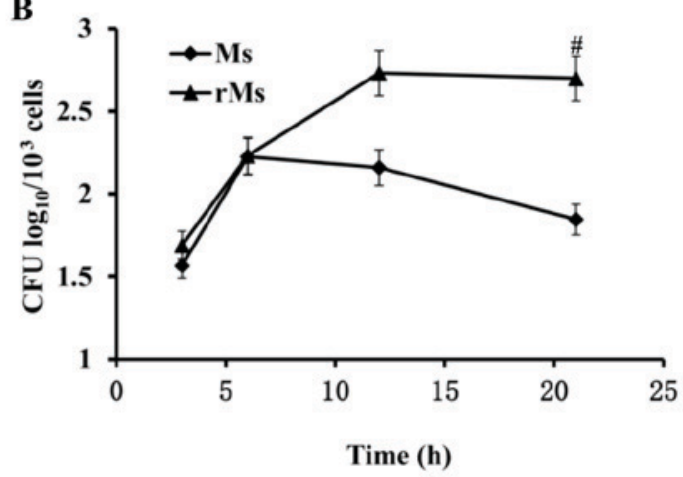

Figure 3. In vitro and ex vivo growth of rMs and Ms. (A) Growth of rMs and Ms was monitored in 3 h intervals by measuring optical density at a wavelength of $600 \mathrm{~nm}$. (B) Growth of rMs and Ms in Raw264.7 murine macrophages. Data are presented as the mean \pm standard deviation. ${ }^{\#} \mathrm{P}<0.05 \mathrm{vs}$. the Ms group. $\mathrm{rMs}$, recombinant Mycobacterium smegmatis.

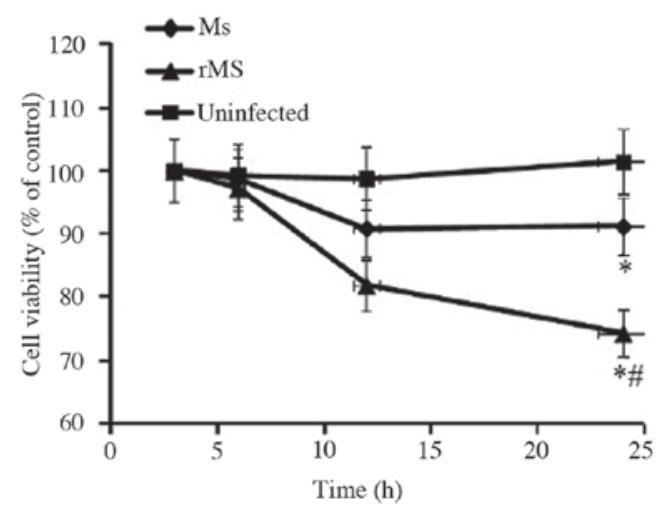

Figure 4. Viability of Raw264.7 murine macrophages analyzed by MTT. The experiment was repeated three times. " $\mathrm{P}<0.05$ vs. the uninfected group; ${ }^{\#} \mathrm{P}<0.05$ vs. the Ms group. rMs, recombinant Mycobacterium smegmatis.

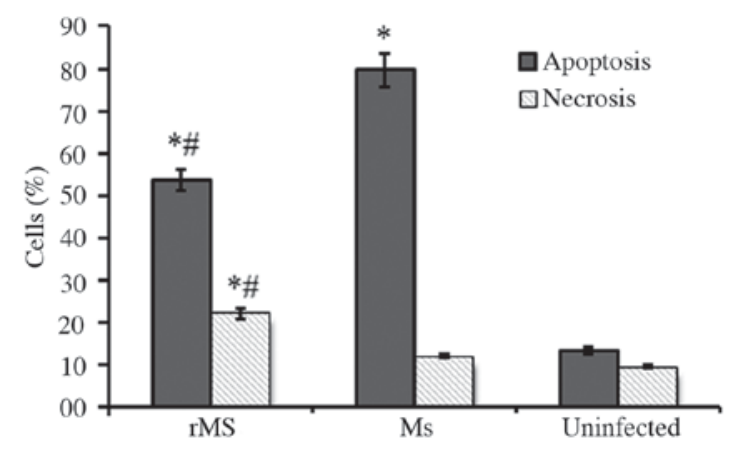

Figure 5. Apoptosis and necrosis of Raw264.7 murine macrophages infected with rMs or Ms. Percentage of apoptotic and necrotic cells was determined using a propidium iodide-conjugated Annexin V and 7-aminoactinomycin D staining and flow cytometry analysis $24 \mathrm{~h}$ following infection. Data are presented as the mean \pm standard deviation. ${ }^{*} \mathrm{P}<0.05$ vs. uninfected; ${ }^{\#} \mathrm{P}<0.05$ vs. Ms. rMs, recombinant Mycobacterium smegmatis.

rMs demonstrated a significant decrease compared with Ms from $12 \mathrm{~h}$ onwards $(\mathrm{P}<0.05$; Fig. 4). The above results indicate that rMs may be more virulent compared with Ms.

Apoptosis and necrosis of macrophages. RAW264.7 murine macrophages infected with $\mathrm{rMs}$ or Ms were stained with PE-conjugated Annexin V and 7AAD, and analyzed by flow cytometry to identified apoptotic and necrotic cells. A total of $24 \mathrm{~h}$ following infection, $50.6 \pm 3.2 \%$ macrophages infected with rMs were apoptotic and $20.7 \pm 2.2 \%$ were necrotic, while $80.2 \pm 4.6 \% \mathrm{Ms}$ infected macrophages were apoptotic and $15.3 \pm 1.4 \%$ were necrotic. Compared with Ms, rMs significantly inhibited apoptosis and induced necrosis of infected macrophages ( $\mathrm{P}<0.05$; Fig. 5$)$. The above results can be associated with expression of Rv2031c-Rv2626c fusion protein by rMS.

Modulatory effects of rMs on the innate immunity of macrophages. To identify factors contributing to the increased survival ability of macrophages infected with rMs, compared with Ms, levels of nitric oxide were measured by Griess assay. NO is a determinant of intracellular bacillary burden in host cells. Following infection, NO was down-regulated in macrophages infected with rMs, compared with macrophages

infected with Ms ( $\mathrm{P}<0.05$; Fig. 6A). Levels of IFN- $\gamma$, IL-6 and cells. Both rMs and Ms inhibited the viability of macrophages $(\mathrm{P}<0.05)$; however, the viability of macrophages infected with
Effect of rMs on viability of macrophages. To determine the effect of rMs on viability of macrophages, MTT analysi was performed at different time points following infection of RAW264.7 murine macrophages with rMs or Ms. A total of $24 \mathrm{~h}$ following infection, the viability of macrophages was equal to $78.8 \pm 3.9 \%$ in cells infected with $\mathrm{rMs}, 90.9 \pm 4.5 \%$ cells infected with Ms and $101.4 \pm 5.1 \%$ in uninfected control 
A

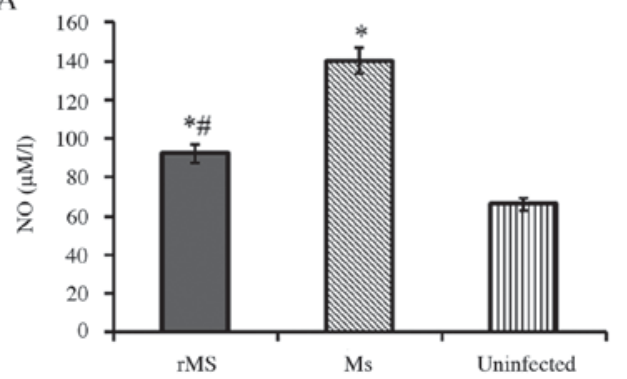

C

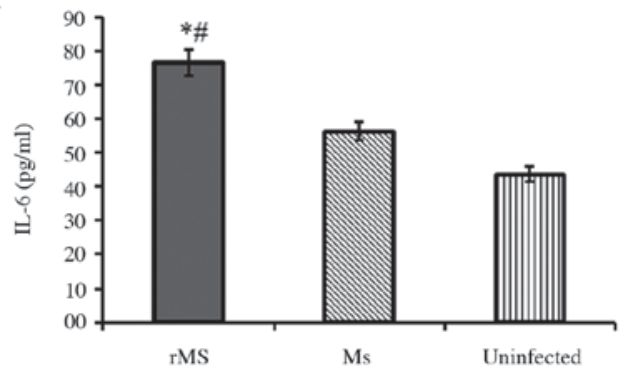

B

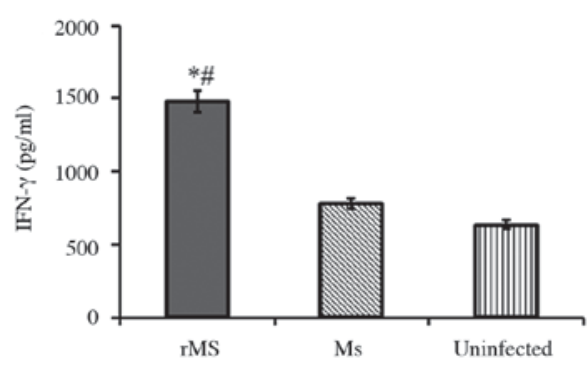

D

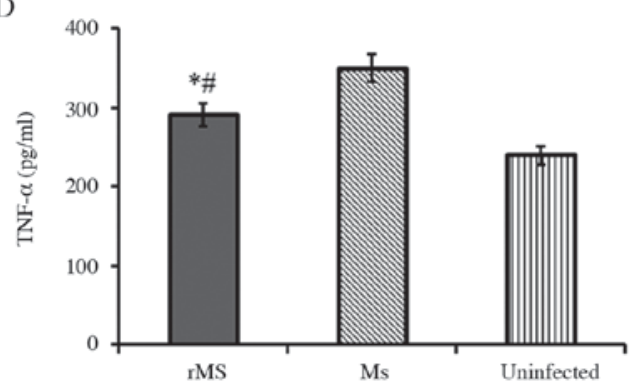

Figure 6. Innate immunity of Raw264.7 murine macrophages infected with rMs or Ms. Levels of (A) NO, (B) IFN- $\gamma$, (C) IL-6 and (D) TNF- $\alpha$ in culture supernatants of infected or uninfected macrophages $24 \mathrm{~h}$ after infection. Data are presented as the mean \pm standard deviation. ${ }^{*} \mathrm{P}<0.05 \mathrm{vs}$. the uninfected group; ${ }^{\#} \mathrm{P}<0.05$ vs. the Ms group. rMs, recombinant Mycobacterium smegmatis. NO, nitric oxide; IFN- $\gamma$, interferon- $\gamma$; IL, interleukin; TNF- $\alpha$, tumor necrosis factor- $\alpha$.

TNF- $\alpha$ in infected macrophages were determined by ELISA. The results demonstrated that secretion of IFN- $\gamma$ and IL-6 in macrophages infected with rMs was significantly up-regulated $(\mathrm{P}<0.05)$, but levels of TNF- $\alpha$ were down-regulated $(\mathrm{P}<0.05)$, compared with macrophages infected with Ms. The above results demonstrate that expression of Rv2031c-Rv2626c fusion protein in rMs can modulate the innate immunity of macrophages infected with rMs to favor intracellular survival of rMs.

\section{Discussion}

It has been demonstrated that DosR regulon, composed of 48 co-regulated genes, is essential for the survival of Mtb in macrophages (10). Dormancy-associated antigens encoded by DosR genes in Mtb serve physiological and immuno-modulatory functions of the host immune system $(11,12)$. In the present study, a fusion protein of dormancy-associated antigens Rv2031c and Rv2626c was expressed in a non-pathogenic strain of Ms. The results of the present study demonstrated that expression of the fusion protein Rv2031c-Rv2626c in rMs prolonged the duration of growth lag-phase of rMs in vitro. The aforementioned data are consistent with a previous report, in which overexpression of Rv2031c in Ms resulted in a significant lag in growth of Ms (5). In order to determine whether expression of Rv2031c-Rv2626c alters the intracellular survival ability of Ms, macrophages were infected with rMs or Ms. Compared with the Ms strain, infectivity of rMs was not affected by expression of the fusion protein Rv2031c-Rv2626c. Virulence and survival of rMs in macrophages were enhanced, the number of viable cells in macrophages infected with rMs was markedly decreased and the number of intracellular rMs bacteria increased. A previous study reported that a Mtb mutant, in which Rv2031c gene was replaced by a hygromycin resistance gene, was attenuated and demonstrated inhibited growth in a macrophage model (7); however, Hu et al (13) reported that increased numbers of CFU were observed in mice or macrophages that they were infected with an unmarked Rv2031c deletion mutant of Mtb when compared with the orginal Mtb strain.

It has been reported that virulent Mtb can inhibit apoptosis and trigger necrosis of host macrophages to evade innate immunity and delay the initiation of adaptive immunity (3). By contrast, attenuated Mtb and non-pathogenic mycobacteria induce apoptosis of macrophages, an innate defense mechanism that reduces bacterial viability $(14,15)$. Therefore, in the present study, apoptosis and necrosis of infected macrophages were observed. The results of the present study demonstrated that, compared with Ms, apoptosis of macrophages infected with rMs was decreased, while necrosis was increased. NO production is an antimicrobial mechanism employed by macrophages (16). In the present study, compared with macrophages infected with Ms, macrophages infected with rMs demonstrated decreased NO levels in macrophages. Therefore, it can be hypothesized that inhibition of NO enhanced the survival of rMs in macrophages.

Macrophages eliminate invading Mtb directly and secrete cytokines to mediate host immune responses (17). The present study investigated secretion of IFN- $\gamma$, TNF- $\alpha$ and IL- 6 from infected macrophages. The results of the present study demonstrated that levels of IFN- $\gamma$ and IL- 6 markedly increased compared with macrophages infected with Ms, while the levels of TNF- $\alpha$ decreased. TNF- $\alpha$ is an extrinsic mediator of apoptosis, which has been demonstrated to have a negative impact on the survival of mycobacteria within macrophages. More virulent strains appear to inhibit expression of TNF- $\alpha$ (18). It can be hypothesized that reduced apoptosis of macrophages 
infected with rMs may be associated with inhibition of expression of TNF- $\alpha$, but the underlying mechanism remains to be elucidated.

In conclusion, the present study demonstrated that expression of the fusion protein of dormancy-associated antigens Rv2031c and Rv2626c in Ms can serve a physiological function of a dormancy-associated antigen. The fusion protein also modulated the innate immunity of host macrophages, favoring intracellular bacillary survival. However, the mechanism underlying intracellular survival mediated by dormancy-associated antigens in Mtb, remain to be elucidated.

\section{Acknowledgements}

The present study was supported by the National Science and Technology Major Project of China (grant no. 2012ZX10003008-007) and the National Natural Science Foundation of China (grant no. 31501112).

\section{References}

1. World Health Organization (WHO): Global Tuberculosis Report WHO, Geneva, pp204, 2015.

2. Gideon HP and Flynn JL: Latent tuberculosis: What the host 'sees'? Immunol Res 50: 202-212, 2011.

3. Liu PT and Modlin RL: Human macrophage host defense against Mycobacterium tuberculosis. Curr Opin Immunol 20: 371-376, 2008.

4. Gerasimova A, Kazakov AE, Arkin AP, Dubchak I and Gelfand MS: Comparative genomics of the dormancy regulons in mycobacteria. J Bacteriol 193: 3446-3452, 2011.

5. Yuan Y, Crane DD and Barry CE III: Stationary phase-associated protein expression in Mycobacterium tuberculosis: function of the mycobacterial alpha-crystallin homolog. J Bacteriol 178: 4484-4492, 1996.

6. de Souza GA1, Arntzen MØ, Fortuin S, Schürch AC, Målen H, McEvoy CR, van Soolingen D, Thiede B, Warren RM and Wiker HG: Proteogenomic analysis of polymorphisms and gene annotation divergences in prokaryotes using aclustered mass spectrometry-friendly database. Mol Cell Proteomics 10 M110.002527, 2011.
7. Yuan Y, Crane DD, Simpson RM, Zhu YQ, Hickey MJ, Sherman DR and Barry CE III: The 16-kDa alpha-crystallin (Acr) protein of Mycobacterium tuberculosis is required for growth in macrophages. Proc Natl Acad Sci USA 95: 9578-9583, 1998.

8. Rosenkrands I, Slayden RA, Crawford J, Aagaard C, Barry CE III and Andersen P: Hypoxic response of Mycobacterium tuberculosis studied by metabolic labeling and proteome analysis of cellular and extracellular proteins. J Bacteriol 184: 3485-3491, 2002.

9. GaoH, Bai Y, Xue Y, Wang L, Fan A, Ding X and Xu Z: Expression, purification, and characterization of soluble RpfD with high bioactivity as a recombinant protein in Mycobacterium vaccae. Protein Expr Purif 55: 112-118, 2007.

10. Boon C and Dick TP: How Mycobacterium tuberculosis goes to sleep: The dormancy survival regulator DosR a decade later. Future Microbiol 7: 513-518, 2012.

11. Singh S, Saraav I and Sharma S: Immunogenic potential of latency associated antigens against Mycobacterium tuberculosis. Vaccine 32: 712-716, 2014.

12. Serra-Vidal MM, Latorre I, Franken KL, Díaz J, de Souza-Galvão ML, Casas I, Maldonado J, Milà C, Solsona J, Jimenez-Fuentes MÁ, et al: Immunogenicity of 60 novel latency-related antigens of Mycobacterium tuberculosis. Front Microbiol 5: 517, 2014.

13. Hu Y, Movahedzadeh F, Stoker NG and Coates AR: Deletion of the Mycobacterium tuberculosis alpha-crystallin-like hspX gene causes increased bacterial growth in vivo. Infect Immun 74: 861-868, 2006.

14. Srinivasan L, Ahlbrand S and Briken V: Interaction of Mycobacterium tuberculosis with host cell death pathways. Cold Spring Harb Perspect Med 4: pii: a022459, 2014.

15. Fratazzi C, Arbeit RD, Carini C, Balcewicz-Sablinska MK, Keane J, Kornfeld $\mathrm{H}$ and Remold HG: Macrophage apoptosis in mycobacterial infections. J Leukoc Biol 66: 763-764, 1999.

16. Ferrari CK, Souto PC, França EL and Honorio-França AC: Oxidative and nitrosative stress on phagocytes' function: From effective defense to immunity evasion mechanisms. Arch Immunol Ther Exp (Warsz) 59: 441-448, 2011.

17. O'Garra A, Redford PS, McNab FW, Bloom CI, Wilkinson RJ and Berry MP: The immune response in tuberculosis. Annu Rev Immunol 31: 475-527, 2013.

18. Beham AW, Puellmann K, Laird R, Fuchs T, Streich R, Breysach C, Raddatz D, Oniga S, Peccerella T, Findeisen P, et al: A TNF-regulated recombinatorial macrophage immune receptor implicated in granuloma formation in tuberculosis. PLoS Pathog 7: e1002375, 2011. 